\title{
Effect of probiotics on giardiasis. Where are we?
}

\author{
Luciana Laranjo Amorim Ventura ${ }^{1}$, Dirce Ribeiro de Oliveira ${ }^{2}$, Maria Aparecida Gomes ${ }^{1}$, \\ Marcia Regina Fantoni Torres ${ }^{3 *}$
}

\begin{abstract}
${ }^{1}$ Department of Parasitology, Institute of Biological Sciences, Federal University of Minas Gerais (UFMG), Belo Horizonte, MG, Brazil, ${ }^{2}$ Department of Basic Life Sciences, Governador Valadares Campus, Federal University of Juiz de Fora, Governador Valadares Campus, MG, Brazil ${ }^{3}$ Department of Pediatrics, Faculty of Medicine, UFMG,
\end{abstract}

Belo Horizonte, $M G$, Brazil

\begin{abstract}
Giardiasis, an intestinal infection caused by Giardia lamblia, was recently included in the 'Neglected Diseases Initiative' by the World Health Organization. Symptomatic patients can develop mild diarrhea up to a severe malabsorption syndrome, and children may show significantly impaired cognitive and physical development. Currently, nitroimidazoles are the main class of antibiotic used to treat giardiasis. Despite the efficacy of these drugs, adverse effects and reported resistance have increased, encouraging studies to identify and develop therapeutic alternatives. In this context, probiotics may represent an attractive option. Probiotics are defined as live microorganisms that, when administered in suitable amounts, confer a health benefit to the host. The use of probiotics in the treatment of parasitosis has been suggested because of its beneficial effects, such as a reduced time of gastrointestinal symptoms and parasite load in animal models and humans. Probiotics have been studied as an alternative treatment for giardiasis. In this review, we evaluated probiotic effectiveness in the treatment and prevention of this disease. Based on the studies examined herein, we conclude that probiotics have a beneficial effect on the modulation of giardiasis.
\end{abstract}

Keywords: Giardia lamblia. Giardiasis. Probiotics/effects/evaluation.

\section{INTRODUCTION}

Giardiasis, an intestinal infection caused by the protozoan Giardia lamblia (synonyms - G. intestinalis and G. duodenalis), is considered the most common human intestinal parasitosis in the world. It is frequent in both developed and developing countries, and is estimated to inflict 280 million symptomatic human infections annually. Due to its clinical and epidemiological importance, in 2004, the World Health Organization (WHO) included giardiasis in the 'Neglected Diseases Initiative' (Savioli, Smith, Thompson, 2006).

In most cases, infection is asymptomatic, and when symptoms occur, the most common manifestations are fatigue, anorexia, belching, vomiting, flatulence, abdominal pain and distension, and steatorrhea. Such

\footnotetext{
*Correspondence: M. R. F. Torres. Departmento de Pediatria, Faculdade de Medicina, Universidade Federal de Minas Gerais, UFMG, Belo Horizonte, MG, Brazil. Av. Professor Alfredo Balena 170, Santa Efigênia, CEP: 30130-109 - Belo Horizonte, MG, Brazil. Tel.: + 5531-3409-9772. E-mail: marcia.fantoni@gmail.com
}

clinical conditions establish an intestinal malabsorption syndrome that can lead to significant weight loss and nutritional deficiencies of the host and, when present in children, may lead to severe growth and cognitive deficits (Matos et al., 2008).

Among the forms of treatment for giardiasis and its symptoms, the use of drugs such as nitroimidazoles, nitrofurans as well as phytotherapics are commonly highlighted. However, due to an increase in resistance to these compounds, there is an urgent call for the development of novel therapeutic strategies to fight the pathogen in more healthy and effective way. Therefore, nutritional interventions and modifications of the intestinal microenvironment through probiotic administration may be an important therapeutic alternative (Iannitti, Palmieri, 2010). In this context, the aim of this revision is to demonstrate the role of probiotics in treating and preventing clinical and experimental giardiasis.

Here, we summarize the experimental and clinical evidence on probiotics, such as Lactobacillus and Saccharomyces boulardii, by providing a comprehensive 
overview of their efficacy as an alternative therapy for giardiasis. Studies published between 2000 and 2016 were chosen through a systematic search using combinations of the keywords 'Giardia lamblia', 'giardiasis', 'probiotics', and 'treatment' in PubMed and Science Direct electronic databases. Clinical and experimental articles that used probiotics as therapeutic agents and were written in the English language have been included.

\section{Giardia and giardiasis}

Giardia exists under two distinct life forms: cyst, the resistant form, responsible for the transmission of the parasite between vertebrate hosts, and trophozoite, the vegetative form, that replicates within the intestinal tract of the hosts and leads to clinical manifestation. Giardia differentiation occurs through the processes of excystation, in which the parasite changes from cyst to trophozoite, and encystment, when it returns to its resistant form(Lauwaet et al., 2007).

Giardia is transmitted through the fecal-oral route, directly - from person to person or indirectly through the ingestion of food or water contaminated with cysts. Infection is restricted to the intestinal lumen, where trophozoites replicate and adhere to the mucosa resulting in enterocytes apoptosis, increased intestinal permeability and nutrient malabsorption (Solaymani-Mohammadi, Singer, 2011) and, consequently, malnutrition, nutritional deficiencies, and cognitive impairment in children and immunodeficient adults (Simsek, Yildeiz-Zeynek, Kurcer, 2004).

The pathophysiology of acute diarrhea in giardiasis implicates increased rates of enterocyte apoptosis, disruption of the intestinal barrier function, activation of host lymphocytes, $\mathrm{CD} 8^{+}$lymphocyte-mediated shortening of brush border microvilli with or without coinciding villous atrophy, crypt hyperplasia, deficiency of disaccharidases, intestinal malabsorption, anion hypersecretion, and increased intestinal transit(Müller, von Allmen, 2005; Buret et al., 2015).

Both innate and adaptive immunity mechanisms are essential for the elimination of the parasite from the intestine. Innate immunity mechanisms are the first line of defense, and among them are the mucus, peristalsis, cytotoxic factors, nitric oxide, microbiota, mast cells, and dendritic cells (Lopez-Romero et al., 2015). Mast cells have the potential to interfere directly or indirectly in the duodenal growth of G. lamblia trophozoites; they are essential to control G. lamblia infection, especially due to the IL-6 synthesis. I1-6 modulates B-cell maturation and the switching to produce IgA, and mediates T-cell differentiation(Lopez-Romero et al., 2015; Merluzzi et al., 2010). Moreover, mast cell degranulation increases smooth muscle contractility and, in synergy with nitric oxide, that relaxes smooth muscle and modulates intestinal motility, induces peristalsis (Li et al., 2007).

Other defense mechanisms arising from local tissue cells are the cytotoxic effect of Paneth cells and its secretion of defensins, and an increase on mucin secretion, which reduces parasite adhesion to intestinal epithelial cells, allowing for the subsequent removal by peristalsis, and thereby reducing the intensity of the infection (Müller, Allmen, 2005). Most experimental model studies suggest that the host's main defense against Giardia is the production of immunoglobulin A(IgA). The mechanisms are not well understood, but are likely to involve 'immune exclusion', immobilization, or detachment of trophozoites from the intestinal epithelium or the mucus layer, rather than direct killing (Eckmann, 2003).

While intestinal antibodies and, more specifically, anti-variant surface protein $\operatorname{IgA}$ antibodies were proven to be involved in modulating antigenic variation of the parasite, the participation of the local antibody response in control of the parasite infection is still controversial (Müller, von Allmen, 2005).

$\mathrm{T}$ cell participation is associated with disease progression, since the depletion of $\mathrm{CD} 4^{+} \mathrm{T}$ cells in animals resulted in chronic giardiasis, whereas $\mathrm{CD} 8^{+} \mathrm{T}$ cells are more related to intestinal mucosa tissue damage resulting from giardiasis than with controlling the infection (Scott, $\mathrm{Yu}$, Buret, 2004). IgA and $\mathrm{CD}^{+} \mathrm{T}$ cells are fundamental to the process of Giardia clearance. It has been suggested that $\mathrm{CD} 4^{+} \mathrm{T}$ cells play a double role during the anti-Giardia immune response. First, they activate and stimulate the differentiation of B cells to generate Giardia-specific antibodies. Second, they act through a B-cell-independent mechanism that is probably mediated by Th17 cells (Lopez-Romero et al., 2015).

\section{Giardia and intestinal microbiome association}

It is widely known that the intestinal microbiota plays a relevant role in the maintenance of human health. This includes impeding colonization by pathogenic agents, fermenting non-soluble fibers, which produce short chain fatty acids, and stimulating immune system development (Sharma, Young, Neu, 2010). The linkages among the human body, gut microbiota, and parasites result in a complex ecosystem where alterations in one of these components determine a counter response in the others (Berrilli et al., 2012).

Torres et al. (2000) showed that Giardia was unable to induce disease in germ-free mice. Furthermore, they 
were the first to demonstrate the stimulatory action of a combination of microbial components isolated from the dominant duodenal microbiota of children with symptomatic giardiasis associated with germ-free mice experimentally inoculated with axenic Giardia lamblia. Nevertheless, none of the combinations of bacteria were able to stimulate the protozoan pathogenicity in germ-free mice in the same level as the microbiota of conventional mice did. These findings can be explained by smaller Peyer's patches, fewer plasma cells, fewer intraepithelial lymphocytes (IELs), impaired antimicrobial peptide and $\operatorname{IgA}$ secretion, and reduced $\mathrm{CD} 4^{+}$and $\mathrm{CD} 8^{+} \mathrm{T}$ cell numbers found in germ-free mice compared to those in conventional mice (Chung et al., 2012).

It has been suggested that the intestinal microbiota composition can potentially interfere in the process of $G$. lamblia infection. Findings from ongoing research indicate that the post-infectious effects of giardiasis may be due to microbiota dysbiosis induced by the parasite during the acute phase of infection (Buret et al., 2015).

Keselman et al. (2016) found that the disruption of the intestinal microbiota through antibiotic treatment for G. lamblia limited the disaccharidase deficiency, prevented pathological $\mathrm{CD} 8^{+} \mathrm{T}$ cell activation, and also did not change the numbers of $\mathrm{CD}^{+} \mathrm{T}$ cells in the lamina propria and the activation of $\mathrm{T}$ cell receptor-expressing intraepithelial lymphocytes (IEL) found in giardiasis. The authors have concluded that differences in the intestinal microbiota can contribute to differences in immune responses and may, therefore, also contribute to differences in clinical outcomes.

\section{Benefits of probiotics in giardiasis}

Giardia is resistant to most currently available drugs (Busatti, Santos, Gomes, 2009). In this sense, new treatment alternatives are needed, aiming not only efficiency, but also a reduction of side effects and treatment resistance.

Probiotics are considered non-pathogenic microorganisms (bacteria or yeasts) that, when consumed in adequate amounts, produce beneficial effects on the health and well-being of the host (Varavallo, Thomé, Teshima, 2008). The competition for binding sites and available food sources in the intestinal lumen limits pathogen survival in this environment (Reid, Hammond, 2005). The most commonly consumed probiotics belong to the genus Lactobacillus and Bifidobacterium. Mechanisms by which probiotics might improve host health include immune function augmentation through reinforcing mucosal barrier function, reducing mucosal transfer of luminal organisms and metabolites to the host, increasing mucosal antibody production, strengthening epithelia integrity and direct antagonism of pathogenic microorganisms (Conlon, Bird, 2015).

Some bacteria genera, such as Lactobacillus and Bifidobacterium, have a direct relation with immune response stimulation because they enhance IgA production and secretion through an alteration of the cytokine milieu in the gut mucosa, induce epithelial cell expression of TGF $\beta$ and IL-10 as well as IL-6, which potentiate IgA production, and induce/augment the expression of polymeric Ig receptors on the basolateral surface of intestinal epithelial cells (Hardy et al., 2013).

However, in order for the desired benefits to be gained, probiotics must be present in functional foods for consumption in adequate amounts. Some studies suggest that the effective therapeutic dose for health benefits is 5 billion colony forming units a day $\left(5 \times 10^{9} \mathrm{CFU} /\right.$ day $)$ for at least five days (Gupta, Garg, 2009).

\section{Experimental trials with probiotics in giardiasis in animal models}

Studies involving the action of probiotics as an alternative therapy on enteric parasites, such as $G$. lamblia, have been conducted due to their worldwide epidemiological importance as well as because of the damage caused upon the host. The first report on the in vivo effect of Lactobacillus johnsonii Lal against G. lamblia (Humen et al., 2005) has demonstrated that animals treated with $10^{8} \mathrm{CFU}$ for seven days before trophozoite inoculation did not show any morphological damage on the intestinal epithelium, and had a reduced proportion of active trophozoite and infection length.

Experimental studies have demonstrated that Giardia infection reduced both the severity and duration when probiotics (Lactobacillus rhamnosus, Lactobacillus casei) were administered prior or simultaneous to the parasite. Daily administration of $10^{9} \mathrm{CFU}$ of Lactobacillus casei in gerbils for 30 days reduces the proportion of active number of trophozoites in the gut and leads to the resolution of a Giardia infection within 14 days, and protected against parasitic-induced mucosal damage, suggesting that probiotics offer a safe and effective mode to prevent and treat Giardia infection (Shukla, Devi, Sehgal, 2008). Normal microvillus morphology and enhanced number of goblet cells were also found in probiotic-fed Giardia-infected mice compared to severe microvillus atrophy, edematous, vacuolated epithelial cells, and ileitis in Giardia-infected mice (Shukla, Sidhu, Verma, 2012). 
Goyal, Tiwari, Shukla (2011) compared the effectiveness of 4 bacterial strains (Lactobacillus rhamnosus GG (LGG), L. acidophilus, L. plantarum and L. casei) in the modulation of murine giardiasis. Animals received a daily, single strain $10^{9} \mathrm{CFU}$ dose via orogastric gavage. LGG has been proven more effective in reducing the G. lamblia cycle duration, for its increased rates of cyst excretion and reduced the number of active trophozoite in the intestine, leading to disease suppression approximately 13 days after experimental inoculation.

Recent studies have suggested that probiotics have an anti-Giardia effect and modulate the mucosal immune response. Goyal, Shukla (2013) showed that oral administration of the probiotic LGG $\left(1 \times 10^{9}\right.$ lactobacilli/ $/ 0.1 \mathrm{~mL}$ ) to Giardia-infected mice with $1 \times 10^{6}$ trophozoites $/ 0.1 \mathrm{~mL}$, restores the normal gut microbiota and modulates the mucosal immune response, since a significant increase in the levels of cytokines IL-6 and IL-10, specific secretory $\operatorname{IgA}$ antibody, $\operatorname{IgA}^{+}$cells, and $\mathrm{CD}^{+} \mathrm{T}$ lymphocytes were observed in contrast with the reduced levels of pro-inflammatory cytokine INF and decreased percentage of cytotoxic $\mathrm{CD} 8^{+} \mathrm{T}$ lymphocytes in the lamina propria. Goyal, Rishi, Shulka (2013) also showed the antioxidative properties of LGG in Giardia mediated tissue injury. Probiotics fed, Giardia-infected mice, showed a significant increase in the levels of antioxidants (reduced glutathione and superoxide dismutase) and intestinal disaccharidases (sucrase and lactase) and decreased levels of oxidants in the small intestine in comparison with Giardia-infected mice. Histopathological findings also revealed an almost normal cellular morphology of the small intestine in probiotic-fed Giardia-infected mice compared with non-probiotic-fed Giardia-infected mice. Fused enterocytes, villous atrophy, and increased infiltration of lymphocytes were observed in Giardia-infected mice, thereby suggesting that the effects of probiotic LGG are biologically plausible and could be used as an alternative microbial interference therapy.

It has been demonstrated the ability of Enterococcus faecium SF68 in the improvement of specific anti-Giardia immune response in mice (Benyacoub et al., 2005) This improvement was characterized by a progressive increase in intestinal $\operatorname{IgA}$ and serum $\operatorname{IgG}$ levels, and consequent decline of the enteric parasitic and antigenic overload. In addition, a higher proportion of $\mathrm{CD}^{+}{ }^{+} \mathrm{T}$ cells in Peyer's patches and spleen could be observed, suggesting that this probiotic might be useful in the treatment of Giardia infection.

Shukla, Kaur, Sharma (2013) also demonstrated the therapeutic effect of probiotic Lactobacillus casei, alone and in conjunction with antiprotozoal drugs like albendazole, tinidazole, metronidazole, and nitazoxanide, on the outcome of giardiasis in murine model. The coadministration of the probiotic and albendazole reduced both the excretion of Giardia cysts in feces and trophozoite counts in the intestinal fluid, and restored villi and crypts to normal morphology. The authors suggested that probiotics and antiprotozoal drugs in combination can be a more effective therapy for improved treatment and enhanced recovery from gastrointestinal diseases.

Other authors evaluated the ability of kefirfermented milk, composed mainly of bacteria and yeasts in a complex symbiotic association, to protect mice from $G$. lamblia infection. They found that feeding mice with kefir reduces infection and promotes the activation of different mechanisms of humoral and cellular immunity that are downregulated by parasitic infection, thus contributing to protection (Franco et al., 2013).

Amer, Mossallan, Mahrous (2014) evaluated the therapeutic efficacy of bacteriocins derived from newly isolated Egyptian strains of probiotics Lactobacilli [L. acidophilus (P106) and L.plantarum (P164)] against $G$. lamblia, both in vitro and in vivo. In vitro results showed that $50 \mu \mathrm{g}$ of L. acidophilus bacteriocin reduced the adherence and the number of trophozoites by $58.3 \pm$ $4.04 \%$. Oral inoculation of $50 \mu \mathrm{g} / \mathrm{mouse}$ L. acidophilus bacteriocin for 5 successive days reduced the intestinal parasite density, and ameliorated intestinal pathology of infected mice. The authors concluded that L.acidophilus (P106) derived bacteriocin should be a great promise as a potential therapeutic safe alternative to existing commercial drugs.

\section{Clinical trials with probiotics in gastrointestinal diseases}

The current literature offers few studies about the use of probiotics as an alternative therapy for giardiasis. However, it is possible to find a greater amount of studies that highlight the benefits of ingesting such microorganisms for the treatment of some enteric diseases with similar symptoms to the ones caused by G. lamblia infection, particularly diarrhea.

The use of a probiotic cannot ignore the knowledge of the genus and species of the strain. In pediatric patients, their role for treating acute viral gastroenteritis and preventing antibiotic-associated diarrhea in healthy children has been demonstrated (Cardile et al., 2016). The risk of infection associated with the use of probiotics is relatively low; however, there are selected groups of patients in who they should be used with caution (Olveira, Gonzalez-Molero, 2016). 
Mansour-Ghanaei et al. (2003) studied patients with acute diarrhea caused by amebiasis, which were treated with antibiotics associated with a $250 \mathrm{mg}$ of $S$. boulardii orally, thrice a day, for 10 days. This joint administration significantly reduced the symptoms' duration and the number of excreted cysts after four weeks. This was associated with the yeast's great potential to restore beneficial effects from the normal intestinal microbiota, although the precise mechanism of action has not been elucidated.

Regarding the beneficial action of S. boulardii associated with antibiotic therapy, Besirbellioglu et al. (2006) have shown yeast's efficiency as an adjunctive therapy on adult patients infected with G. lamblia and treated with metronidazole $750 \mathrm{mg}, 3$ times daily, along with probiotic dosage of $250 \mathrm{mg}$, administered twice daily, for 10 days. Authors has hypothesized that this yeast exerts a trophic effect in the small intestine, increases levels of enteric disaccharidases, stimulates intestinal immune system, and changes intestinal enzyme activation.

Working with children who had persistent diarrhea, Gaon et al., (2003) showed that the administration of $S$. boulardii as well as combined administration of different L. casei strains with L. acidophillus (Cerela strain) significantly reduced length and frequency of diarrhea and gastrointestinal symptoms such as vomiting, abdominal distention, and colic at any time during the treatment period. Both treatments administered $10^{10}$ to $10^{12} \mathrm{CFU} / \mathrm{g}$ of probiotic in a $175 \mathrm{~g}$ of diet, twice a day, for a 5-day period.

Patel, DuPont (2015) showed that prebiotics, probiotics, and symbiotics are appealing as preventive and therapeutic agents for human medical disorders. Their efficacy depends on the etiology of the disease and the probiotic strain. Restoring the balance of intestinal flora by introducing probiotics for disease prevention and treatment could be beneficial to human health.

\section{CONCLUSIONS}

Probiotics have a positive action in the treatment of enteric parasite infections, and this benefit may be due to several factors, such as change of intestinal microbiota composition, modulation of immune response, and reduction of host-pathogen interactions. However, the exact mechanism by which probiotics modulate Giardia infection requires thorough investigation. We also strongly support more research on the best probiotic or probiotic association to use in giardiasis.

\section{REFERENCES}

Amer EI, Mossallam SF, Mahrous H. Therapeutic enhancement of newly derived bacteriocins against Giardia lamblia. Exp Parasitol. 2014;146:52-63.

Benyacoub J, Pérez PF, Rochat F, Saudan KY, Reuteler G, Antille N, Humen M, De Antoni GL, Cavadini C, Blum S, Schiffrin EJ. Enterococcus faecium SF68 enhances the immune response to Giardia intestinalis in mice. J Nutr. 2005;135(5):1171-1176.

Berrilli F, Di Cave D, Cavallero S, D'amelio S. Interactions between parasites and microbial communities in the human gut. Front Cell Infect Microbiol. 2012;2:141.

Besirbellioglu BA, Ulcay A, Can M, Erlen, H, Tanyuksel, M, Avci, I Araz E, Pahsa A. Saccharomyces boulardii and infection due to Giardia lamblia. Scand J Infect Dis. 2006;38(6-7):479-481.

Buret GA, Amat CB, Manko A, Beatty JK, Halliez MCM, Bhargava A, Motta J P, Cotton J. Giardia duodenalis: New research developments in pathophysiology, pathogenesis, and virulence factors. Curr Trop Med Rep. 2015;2(3):110-118.

Busatti H, Santos JFG, Gomes MA. The old and new therapeutic approaches to the treatment of Giardiasis: Where are we? Biol Targets Ther. 2009;3:273-87.

Cardile S, Alterio T, Arrigo T, Salpietro C. Role of prebiotics and probiotics in pediatric diseases. Minerva Pediatr. 2016;68(6):487-497.

Chung H, Pamp SJ, Hill JA, Surana NK, Edelman SM, Troy EB, et al. Gut immune maturation depends on colonization with a host-specific microbiota. Cell. 2012;149(7):1578-1593.

Conlon MA, Bird AR. The impact of diet and lifestyle on gut microbiota and human health. Nutrients. 2015;7(1): 17-44.

Eckmann L. Mucosal defenses against Giardia. Parasite Immunol. 2003;25(5):259-270.

Franco Mc, Golowczyc Ma, De Antoni Gl, Pérez Pf, Humen M, Serradell M L. Administration of kefir-fermented milk protects mice against Giardia intestinalis infection. J Med Microbiol. 2013;62(12):1815-22.

Gaon D, Garcia H, Winter L, Rodriguez N, Quintas R, Gonzalez SN, Oliver G. Effect of Lactobacillus strains and Saccharomyces boulardii on persistent diarrhea in children. Medicina (B Aires). 2003;63(4):293-298. 
Goyal N, Rishi P, Shukla G. Lactobacillus rhamnosus GG antagonizes Giardia intestinalis induced oxidative stress and intestinal disaccharidases: an experimental study. World J Microbiol Biotechnol. 2013;29(6):1049-1057.

Goyal N, Shukla G. Probiotic Lactobacillus rhamnosus GG modulates the mucosal immune response in Giardia intestinalis - infected BALB/c mice. Dig Dis Sci. 2013;58(5):1218-1225.

Goyal N, Tiwari RP, Shukla G. Lactobacillus rhamnosus GG as an effective probiotic for murine Giardiasis. Interdiscip Perspect Infect Dis. 2011;2011:795219.

Gupta V, Garg R. Probiotics. Indian J Med Microbiol. 2009;27(3):202-209.

Hardy H, Harris J, Lyon E, Beal J, Foey AD. Probiotics, prebiotics and immunomodulation of gut mucosal defenses: homeostasis and immunopathology. Nutrients. 2013;5(6):18691912.

Humen MA, De Antoni GL, Benyacoub J, Costas ME, Cardozo MI, Kozubsky L, et al. Lactobacillus johnsonii La1 antagonizes Giardia intestinalis in vivo. Infect Immun. 2005;73(2):12651269.

Iannitti T, Palmieri B. Therapeutical use of probiotic formulations in clinical practice. Clin Nutr. 2010;29(6):701-725.

Keselman A, Li E, Maloney J, Singer Sm. The Microbiota Contributes to $\mathrm{CD} 8+\mathrm{T}$ cell activation and nutrient malabsorption following intestinal infection with Giardia duodenalis. Infection Imunn. 2016;84(10):2853-2860.

Lauwaet T, Davids BJ, Reiner DS, Gillin FD. Encystation of Giardia lamblia: A model for other parasites. Curr Opin Microbiol. 2007;10(6):554-559.

Li E, Zhao A, Shea-Donohue T, Singer SM. Mast cell-mediated changes in smooth muscle contractility during mouse Giardiasis. Infect Immun. 2007;75(9):4514- 4518.

Lopez-Romero G, Quintero J, Astiazarán-García H, Velazquez C. Host defences against Giardia lamblia. Parasite Immunol. 2015;37(8):394-406.

Mansour-Ghanaei F, Dehbashi N, Yazdanparast K, Shafaghi A. Efficacy of Saccharomyces boulardii with antibiotics in acute amoebiasis. World J Gastroenterol. 2003;9(8):1832-1833.
Matos SM, Assis AM, Prado MDA S, Strina A, Santos LA, Jesus SR, Barreto ML. Giardia duodenalis infection and anthropometric status in preschoolers in Salvador, Bahia State, Brazil. Cad Saúde Públ. 2008;24(7):1527-1535.

Merluzzi S, Frossi B, Gri G, Parusso S, Tripodo C, Pucillo C. Mast cells enhance proliferation of B-lymphocytes and drive their differentiation toward IgA secreting plasma cells. Blood. 2010;115(14):2810-2817.

Müller N, Von Allmen N. Recent insights into the mucosal reactions associated with Giardia lamblia infections. Int $\mathrm{J}$ Parasitol. 2005;35(13):1339-1347.

Olveira G, González-Molero I. An update on probiotics, prebiotics and symbiotics in clinical nutrition. Endocrinol Nutr. 2016;63(9):482-494.

Patel R, Dupont HL. New approaches for bacteriotherapy: prebiotics, new-generation probiotics, and symbiotics. Clin Infect Dis. 2015;60(Suppl 2):S108-121.

Reid G, Hammond JA. Probiotics. Some evidence of their effectiveness. Can Fam Physic. 2005;51(11):1487-1493.

Savioli L, Smith H, Thompson A. Giardia and Cryptosporidium join the 'Neglected Diseases Initiative'. Trends Parasitol. 2006;22(5):203-208.

Scott KG, Yu LC, Buret AG. Role of $\mathrm{CD}^{+}$and $\mathrm{CD} 4^{+} \mathrm{T}$ lymphocytes in jejunal mucosal injury during murine Giardiasis. Infect Immun. 2004;72(6):3536-3542.

Sharma R, Young C, Neu J. Molecular modulation of intestinal epithelial barrier: contribution of microbiota. J Biomed Biotechnol. 2010;2010:305879.

Shukla G, Devi P, Sehgal R. Effect of Lactobacillus casei as a probiotic on modulation of Giardiasis. Dig Dis Sci. 2008;53(10):2671-2679.

Shukla G, Kaur H, Sharma L. Comparative therapeutic effect of probiotic Lactobacillus casei alone and in conjunction with antiprotozoal drugs in murine Giardiasis. Parasitol Res. 2013;112(6):2143-2149.

Shukla G, Sidhu RK, Verma A. Restoration of anthropometric, biochemical and histopathological alterations by Lactobacillus casei supplementation in Giardia intestinalis infected renourished BALB/c mice. Antonie Van Leeuwenhoek. 2012;102(1):61-72. 
Effect of probiotics on giardiasis. Where are we?

Simsek Z, Yildeiz-Zeynek F, Kurcer MA. Effect of Giardia infection on growth and psychomotor development of children aged 0-5 years. J Trop Pediatr. 2004;50(2):90-93.

Solaymani-Mohammadi S, Singer SM. Host immunity and pathogen strain contribute to intestinal disaccharidase impairment following gut infection. J Immunol. 2011;187(7):3769-3775.

Torres, MF, Uetanabaro AP, Costa Af, Alves, CA, Farias, LM, Bambirra, EA, Nicoli, J. Influence of bacteria from the duodenal microbiota of patients with symptomatic Giardiasis on the pathogenicity of Giardia duodenalis in gnotoxenic mice. J Med Microbiol. 2000;49(3):209-215.
Varavallo MA, Thomé JN, Teshima E. Aplicação de bactérias probióticas para profilaxia e tratamento de doenças gastrointestinais. Semina Ciênc Biol Saúde. 2008;29(1):83-104.

Received for publication on $21^{\text {st }}$ June 2017 Accepted for publication on $29^{\text {th }}$ November 2017 